\title{
mecanismo quínion de la formación del color en los ladrillos
}

\section{1. introducción}

1.1. Son frecuentes las consultas de los ceramistas accrca de temas relacionados con el color de sus productos. La complejidad de los fenómenos que dan lugar a la formación del color impide, en la generalidad de los casos, que las soluciones sean sencillas y económicas.

1.2. Cuando hablemos del color de los ladrillos nos referiremos al color de la masa de los ladrillos sin defectos. Los defectos se tratarán como consecuencias de algunas reacciones, cuando se vean éstas.

1.3. El color dependerá, en cada caso, de la composición química de la mezcla cocida, pero composiciones químicas similares pueden dar lugar a coloraciones completamente distintas, si el proceso de fabricación lo es. Estudiaremos la coloración en función de la composición química y de las reacciones que se desarrollen, intentando dar una idea de conjunto de los aspectos principales del tema.

\section{2. composición tipo de las arcillas}

2.1. La composición de las arcillas es muy variada cuantitativamente, pero cualitativamente puede reducirse a muy pocos óxidos. Hedin la reduce a los óxidos de aluminio, de calcio, de silicio y de hierro, cubriendo el aspecto cuantitativo por medio de mezclas de estos óxidos en distintas proporciones.

La elección de esta composición ha sido el resultado de un estudio metódico de numerosos análisis.

2.2. Normalmente aparecen otros elementos en las arcillas, pero en cantidades despreciables desde el punto de vista del color. Estas trazas de elementos pueden causar defectos o coloraciones imprevistas, mas la aparición de este tipo de defectos no es frecuente y normalmente no constituye problemas en la fabricación.

2.3. Las previsiones de colores deben hacerse fundamentándose en las proporciones relativas de los cuatro óxidos mencionados. Hedin ha hecho un estudio muy completo del tema, resumido en la Tabla I, que representa una gran ayuda cuando se trata de predecir coloraciones o calcular correcciones del color. 


\section{3. mecanismo físico de la producción del color}

3.1. El color se produce porque la energía necesaria para ciertos saltos electrónicos se absorbe de las ondas luminosas y se desprende en forma de fotones, con un cierto retraso, al verificarse el salto electrónico contrario. Este fenómeno produce interferencias en ciertas longitudes de onda, particulares para cada elemento o combinación de elementos, en las que se manifiesta la absorción luminosa. Estos salíos electrónicos a los que hacemos referencia sólo son posibles en los elementos de transición, en los que la superposición de los niveles energéticos los permite; en otras palabras: únicamente los elementos de transición son productores de color por ellos mismos.

3.2. Los elementos de transición ocupan en el sistema periódico - tabla larga de Mendelejeff - los grupos del 3 al 8. De todos los elementos que componen estos grupos sólo el hierro se encuentra normalmente en la composición de las arcillas. A veces aparece manganeso, que puede producir coloraciones oscuras, pero su presencia y el desarrollo de sus colores no son normales.

TABLA I

\begin{tabular}{|c|c|c|c|c|c|c|c|}
\hline hajo & $\begin{array}{l}1,175 \\
12010\end{array}$ & 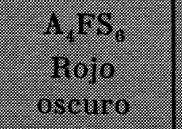 & 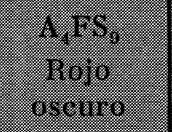 & $\begin{array}{l}\text { A. Fis } \\
\text { Rolo } \\
\text { asciano }\end{array}$ & 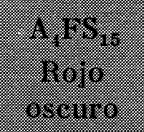 & 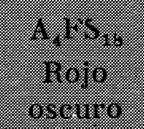 & 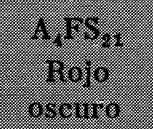 \\
\hline$\frac{64.5}{19.10}$ & 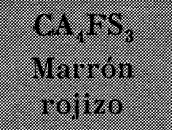 & 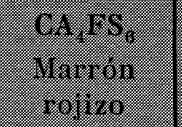 & 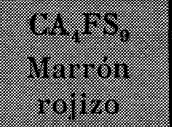 & 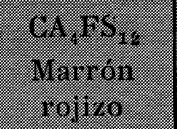 & 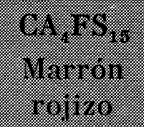 & $\frac{0.1 .15}{1.010}$ & 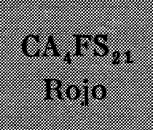 \\
\hline$(4,4.1 \%$ & $\mathrm{C}_{1} \mathrm{~A}_{1}, \mathrm{IS} \mathrm{S}_{3}$ & C., 1.175 & C. 4.105 & C. A, FS. & $(4,1,14.5$ & C. A. IVS. & $0,1,178$, \\
\hline $\begin{array}{l}\text { Pojo } \\
\text { amain } 110\end{array}$ & aharillento & 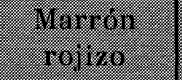 & intail 110 & (inatilis & Amatilitio & a. & ing \\
\hline Amarillo & 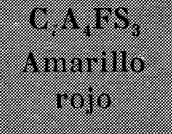 & 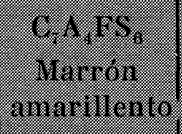 & 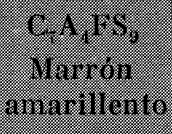 & 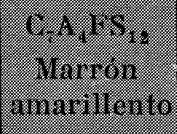 & 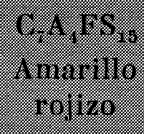 & $\begin{array}{c}\text { C. A. FS } \\
\text { A.marilio } \\
\text { rojolo }\end{array}$ & $\begin{array}{l}\text { C. A I TS } \\
\text { Amarilio } \\
\text { rojo }\end{array}$ \\
\hline
\end{tabular}

3.3. Aunque el hierro es el único agente colorante que se encuentra normalmente en las arcillas no es de extrañar que los colores que se produzcan sean muy variados, pues el color depende fundamentalmente de la cualidad y cantidad de los saltos electrónicos, y éstos, de la situación de los electrones capaces de verificarlos, es decir, del estado de valencia, del número y cualidad de los elementos coordinados, etc. Esta dependencia no se manifiesta únicamente en los compuestos auténticos, sino también en los estados metastables, en las soluciones sólidas y en todos los casos de inter-influencia.

\section{4. mecanismo químico de la producción del color}

4.1. La disposición de los átomos no se verifica al azar, sino que es consecuencia directa de las condiciones en que se desarrolla la reacción. 
Este mecanismo secundario es el que nos interesa a nosotros, ya que, a través de él, podremos influir en el mecanismo primario al que antes hacíamos referencia. Son posibles tantos colores, producidos por un mismo elemento, como "compuestos" finales sean posibles. (Al referirnos a "compuestos" queremos decir cualquier situación en la que dos átomos distintos puedan ejercer influencia mutua, prescindiendo en absoluto del tipo de fuerzas que les unan).

Un mismo elemento de transición no solamente podrá dar compuestos coloreados de modo distinto por separado, sino también mezclas de estos compuestos que tengan "colores suma".

4.2. Toda esta teoría de la formación de compuestos se ve complicada por un hecho fundamental: generalmente en cerámica industrial no se completan las reacciones. Los procesos de fabricación se detienen cuando se han adquirido ciertas propiedades, no siendo normalmente su final un estado de equilibrio.

4.3. Hace unos años se creía que todas las reacciones cerámicas se desarrollaban con una fase líquida intermedia que facilitaba el transporte de los iones o átomos de un compuesto a otro. Hoy se sabe que muchas reacciones ocurren en estado sólido, sin el concurso de ninguna fase líquida. Estas reacciones se desarrollan normalmente a temperaturas inferiores a las de fusión, son más lentas, pero igualmente eficaces que las reacciones en fase líquida.

Estas reacciones consisten en una emigración de átomos o iones, en direcciones distintas, que tienden a hacer de la masa reacciosante un todo homogéneo.

Los mecanismos actualmente admitidos son: la difusión de materia "a lo largo" de la superficie de los cristales o por las superficies de contacto entre cristales distintos y la difusión a través de todo el volumen del cristal, necesariamente más lenta que la anterior, que son las típicas reacciones en estado sólido. El concurso de un flujo plástico en micro-escala y la actuación de determinados agentes, llamados en la bibliografía "dispersión carrier", que ayudan al transporte de materia, no son del todo características reacciones en estado sólido.

En los dos primeros mecanismos la superficie juega un papel importantísimo; la velocidad de reacción en estado sólido depende de la superficie activa de los compuestos reaccionantes.

Esta forma de considerar las reacciones como una respuesta a la solicitación de alcanzar la mayor homogeneidad posible - el mayor desorden, la mayor entropía- puede llevarnos a la consideración de que la creación de fuerzas de enlace entre partículas distintas sólo es parte de esta respuesta, como una trazsmisión de parte de la energía interna que adquieren las pequeñas moléculas con la temperatura, al exterior.

4.4. Existe la teoría de que entre las partículas de un sólido pulverulento existen fuerzas de enlace que dependen de la magnitud de las superficies realmente puestas en contacto. Los gases o líquidos absorbidos modifican estas fuerzas, ocupando superficie, y por esto su expulsión las aumenta, pudiendo llegar a hacer de un pseudo-flúido un sólido real. Cuanto más finas sean las partículas, más ligadas estarán entre sí. El prensado no hace más que aumentar este número de enlaces, almacenándose la energía aplicada en ellos.

El caso de las arcillas en estado plástico deberá explicarse de modo distinto, considerando que la plasticidad es la propiedad de tender hacia un número limitado de enlaces, 
sean del tipo que sean, a través de una o varias moléculas de agua o directamente entre partículas, siendo estados de no equilibrio tanto aquellos en los que el número es mayor como en los que es menor. Una arcilla en estado plástico tenderá a reestablecer los enlaces rotos y se pega consigo misma, pero no adquiere rigidez. La pérdida de la plasticidad en el secado no es más que la consecuencia del aumento del número de enlaces que se produce con la pérdida de agua.

Aparte esta disgresión, cualquiera de los mecanismos antes citados, que se desarrollan durante un proceso a alta temperatura, aumenta el número de enlaces o, lo que es lo mismo, las fuerzas de unión; así se produce la sinterización, sin el concurso de fase líquida alguna.

4.5. A pesar de todo, las reacciones puramente en estado sólido son raras cuando se trata con productos naturales, y lo normal es que si se verifica una determinación cuidadosa se encuentren trazas de fases líquidas.

Las fases gaseosas están normalmente presentes debido a que en el curso de la cocción se producen descomposiciones minerales en el sentido:

$$
\text { sólido }=\text { gas }+ \text { sólido }
$$

Los mecanismos de la sinterización se representan esquemáticamente en la figura 1.

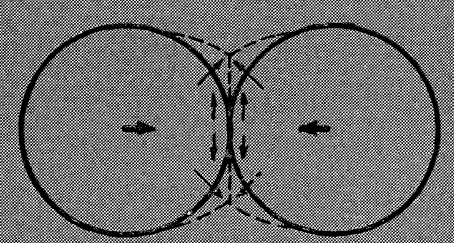

(1)

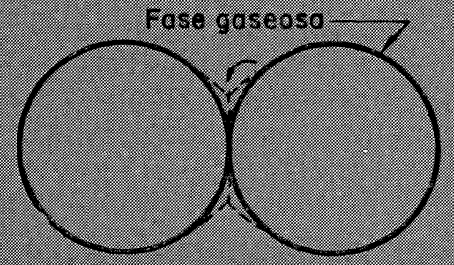

(c)

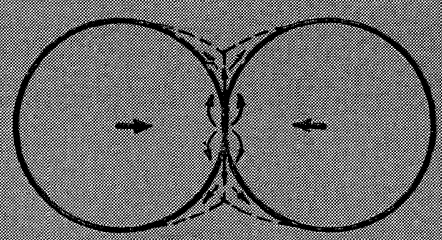

(6)

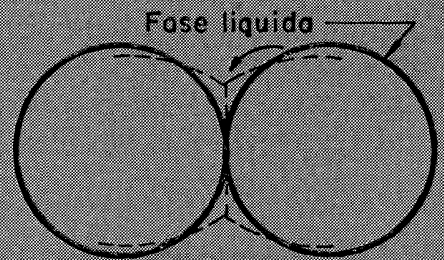

(6.)

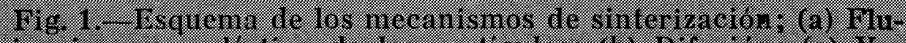
jo víscosso o plístíco de las particulas, (i) Difisión. (c) Vas promización y condensacion? (d) Solución y prectpitación.

4.6. Las reacciones que se desarrollan no sólo producen el aumento de cohesión, sino también los compuestos coloreados. El color producido dependerá de todas las variables que intervienen en el desarrollo de las reacciones: finura, temperatura, tiempo y compuestos reaccionantes.

4.7. Por último, existe el efecto físico de la dilución. Cuanto menos agente colorante haya en una masa, más débil será el color producido. Este efecto, que ha sido discutido, es para nosotros claro, y encontramos una comprobación de este hecho en la formación de colores amarillos a la que más adelante haremos referencia. 


\section{5. los colores producidos por el hierro}

5.1. El color depende, fundamentalmente, de la forma de entrar en la composición de la arcilla; se presenta como:

Oxido:

$\mathrm{FeO}$

$\mathrm{Fe}_{2} \mathrm{O}_{3}$ (Hematites)

$\mathrm{Fe}_{3} \mathrm{O}_{4}$ (Magnetita)

Sulfuro:

$\mathrm{S}_{2} \mathrm{Fe}$ (Pirita)

Carbonato:

$\mathrm{CO}_{3} \mathrm{Fe}$ (Siderosa)

Sulfatos:

$\mathrm{SO}_{4} \mathrm{Fe}$

$\left(\mathrm{SO}_{4}\right)_{3} \mathrm{Fe}_{2}$

Están relacionados en el orden de frecuencia.

Junto con esta composición mineral, la atmósfera y la temperatura nos determinarán el color producido. La intensidad vendrá determinada por la finura y el tiempo.

Trataremos de estudiar los fenómenos producidos según el orden en que ocurren durante la cocción, aunque en muchos casos se originen reacciones en intervalos de temperatura que se solapan.

5.2. La primera fase de la cocción es una deshidratación que afecta al agua residual del amasado y a la llamada "agua de hidratación", que se pierde de forma continua entre $\operatorname{los} 150^{\circ} \mathrm{C}$ y una temperatura nunca superior a los $400^{\circ} \mathrm{C}$. En este espacio de temperaturas se pierde además el agua de cristalización, que es una reacción endotérmica detectable por ATD.

5.3. Inmediatamente se produce la descomposición de los sulfuros —piritas_, que si transcurre en atmósfera oxidante sigue los caminos:

$$
\begin{aligned}
& 4 \mathrm{FeS}_{2}+11 \mathrm{O}_{2}=2 \mathrm{Fe}_{2} \mathrm{O}_{3}+8 \mathrm{SO}_{2} \\
& 4 \mathrm{FeS}_{2}+15 \mathrm{O}_{2}=2 \mathrm{Fe}_{2} \mathrm{O}_{3}+8 \mathrm{SO}_{3}
\end{aligned}
$$

Estas reacciones comienzan sobre los $425^{\circ} \mathrm{C}$ y a los $520^{\circ} \mathrm{C}$ están normalmente terminadas.

En atmósfera reductora, a la misma temperatura aproximadamente,

$$
2 \mathrm{FeS}_{2}=\mathrm{S}_{2}+2 \mathrm{SFe},
$$

- el vapor de azufre puede combinarse con oxígeno de la atmósfera del horno haciéndola más reductora, pudiendo también dar lugar a sulfatos alcalinos o alcalinotérreos, que debilitan o enmascaran el color por la posterior aparición de eflorescencias.

5.4. El sulfuro ferroso que queda en los ladrillos puede reaccionar con oxígeno dando productos distintos, según la cantidad relativa de éste: 


$$
\begin{aligned}
& 6 \mathrm{FeS}+2 \mathrm{O}_{2}=\mathrm{SO}_{4} \mathrm{Fe} \\
& 6 \mathrm{FeS}+13 \mathrm{O}_{2}=2 \mathrm{Fe}_{2}\left(\mathrm{SO}_{4}\right)_{3}+2 \mathrm{FeO} \\
& 4 \mathrm{FeS}+7 \mathrm{O}_{2}=2 \mathrm{Fe}_{2} \mathrm{O}_{3}+4 \mathrm{SO}_{2} \\
& 4 \mathrm{FeS}+9 \mathrm{O}_{2}=2 \mathrm{Fe}_{2} \mathrm{O}_{3}+4 \mathrm{SO}_{3}
\end{aligned}
$$

Los sulfatos producidos se descomponen como se indica más adelante.

5.5. El sulfuro ferroso es lo suficientemente estable como para formarse por encima de $\operatorname{los} 500^{\circ} \mathrm{C}$, combinándose fácilmente con ciertos silicatos, dando un vidrio viscoso y negro - o verde muy oscuro - que contribuye a la formación del defecto conocido como "corazón negro" (black core). Si este vidrio encierra partes de sulfuro ferroso sin reaccionar, y el calentamiento prosigue hasta que se verifica la descomposición, lo que ocurre normalmente, se producen hinchamientos y roturas. La formación de estos defectos depende en gran parte de la permeabilidad de los materiales en verde. Las granulometrías más finas facilitan la eliminación de los sulfuros y de la materia orgánica o carbonosa, protagonistas de la formación del corazón negro. Parece ser imprescindible para la formación de este defecto la atmósfera reductora, en la que no se produce la oxidación de los compuestos ferrosos y el carbono se elimina con dificultad.

5.6. En los hornos en los que el control de la atmósfera no es perfecto es fácil que una corriente de gases reductores incida sobre una parte de la carga, dando lugar a que en esta zona se desarrollen las relaciones de modo distinto, produciendo extensas manchas de color oscuro que a veces afectan a gran parte de la carga del horno. Esta influencia de las atmósferas reductoras, sobre todo a las temperaturas críticas más bajas, se usa en ocasiones para obtener ladrillos de coloración oscura por medio de la operación llamada "flashing", que consiste en disminuir la entrada de aire del horno.

5.7. Prácticamente superpuesta con esta reacción ocurre la descomposición del carbonato ferroso que, salvo en el desprendimiento de carbónico, que no afecta para nada al color, se comporta como óxido ferroso, haciendo la salvedad de que puede producirse una total o parcial evolución hacia férrico en el caso de ser la atmósfera oxidante.

5.8. La descomposición de los sulfatos comienza a una temperatura bastante indeterminada y acaba sobre $\operatorname{los} 600^{\circ} \mathrm{C}$, incluso en el caso de verificarse el calentamiento de forma rápida. Al final de la cocción las mezclas que contenían sulfato ferroso suelen tener color amarillo oscuro, aunque puede obtenerse toda una gama de colores y sombras, según las condiciones de la cocción. El sulfato férrico da normalmente coloraciones rojas. La atmósfera en que se verifica la descomposición tiene una influencia definitiva sobre el color obtenido; la atmósfera oxidante oscurece el color final en el primer caso y refuerza el rojo en el segundo. La reductora purifica el amarillo en el primer caso y hace tender el rojo hacia tonos más oscuros en el segundo. Todo esto es la consecuencia de que un mismo elemento en dos grados de valencia distintos produce colores más oscuros que cada grado de valencia por separado.

5.9. Si, por cualquier circunstancia, la descomposición de los sulfatos se produce después de que ha comenzado el proceso de vitrificación, se originan hinchamientos y en determinados casos roturas o desperfectos debidos al afloramiento de las burbujas de gas producidas. De todos modos la presencia de sulfatos de hierro en las arcillas es poco frecuente. La descomposición da como productos finales los óxidos. 
5.10. Todas estas reacciones son la primera fase de la verdadera cocción, o la segunda del proceso completo. Las temperaturas a las que se producen son demasiado bajas para que hayan comenzado los procesos de sinterización y las reacciones en estado sólido. Al final de esta reacción los compuestos están como óxidos o silicatos; compuestos muy estables, que no se descomponen hasta temperaturas mucho más altas. El aumento de la temperatura ocasiona un aumento de la reactividad de los compuestos presentes que se comportan como óxidos ácidos y básicos activos, combinándose entre sí para dar "sales" más estables aún. Esta tendencia al equilibrio es aquella solicitación de homogeneidad de la que hablábamos antes; ésta es la diferencia de potencial que determina la producción de las reacciones en estado sólido - verdaderas emigraciones de materia - y de la sinterización.

\section{6. final de la cocción, reacciones entre óxidos.-colores producidos por los óxidos de hierro}

6.1. La forma de presentarse los óxidos depende de su historia; y de esta forma de presentación depende el color que desarrollan, si otra circunstancia no ha determinado ya una evolución definitiva, corazón rosa, corazón negro, manchas rojas o negras, etc.

6.2. Las reacciones son múltiples, pero pueden agruparse en unas pocas clases:

$\left.1 .^{\circ}\right)$ Formación de soluciones sólidas, como las de hematita en óxido cálcico, de color amarillo, que puede ser el típico ejemplo de dilución del color. Dal y Hedin opinan, sin embargo, que los colores amarillos no se forman sin la presencia de alúmina, que podría actuar en este caso como inhibidor de la reacción de formación de ferrito cálcico. Las soluciones sólidas de hematites en alúmina son rojas; y en casos en los que no exista una fuerte preponderancia de óxido cálcico puede ser éste el mecanismo de formación de color.

$2^{\circ}$ ) Reacciones en estado sólido, o que así se consideran, como las de formación de ferrito cálcico, que se desarrolla en atmósfera reductora dando colores grises. Este color es muy parecido al que se obtiene cuando el óxido férrico se reduce parcialmente a ferroso o viceversa.

A baja temperatura el óxido férrico puede reaccionar con los silicatos, dando compuestos altamente coloreados. Cuando este fenómeno se produce en forma discontinua, de gránulos aislados, produce un defecto muy típico llamado manchas rosa (pink spot). Esta misma reacción es la responsable del defecto llamado corazón rosa (pink core), cuando la reacción está impedida superficialmente por cualquier causa, como que la atmósfera sea reductora. Un efecto parecido se produce en las arcillas ricas en sulfatos alcalinos o alcalinotérreos, en las que durante la última fase de secado y primera de la cocción se origina una emigración hacia la superficie de las sales solubles reforzándose el color en el interior y debilitándose en la superficie.

6.3. A partir de los $1.100^{\circ} \mathrm{C}$ el óxido férrico se descompone, dependiendo la temperatura de descomposición de los demás constituyentes de la mezcla. Si esta descomposición se produce después de comenzada la vitrificación, que normalmente a esta temperatura ya está bastante avanzada, se producen fuertes hinchamientos, ya que el $\mathrm{Fe}_{2} \mathrm{O}_{3}$ origina un volumen de gas casi de 300 veces su volumen inicial. Para evitar este inconveniente es necesario que el hierro esté reducido antes de comenzar la vitrificación. 


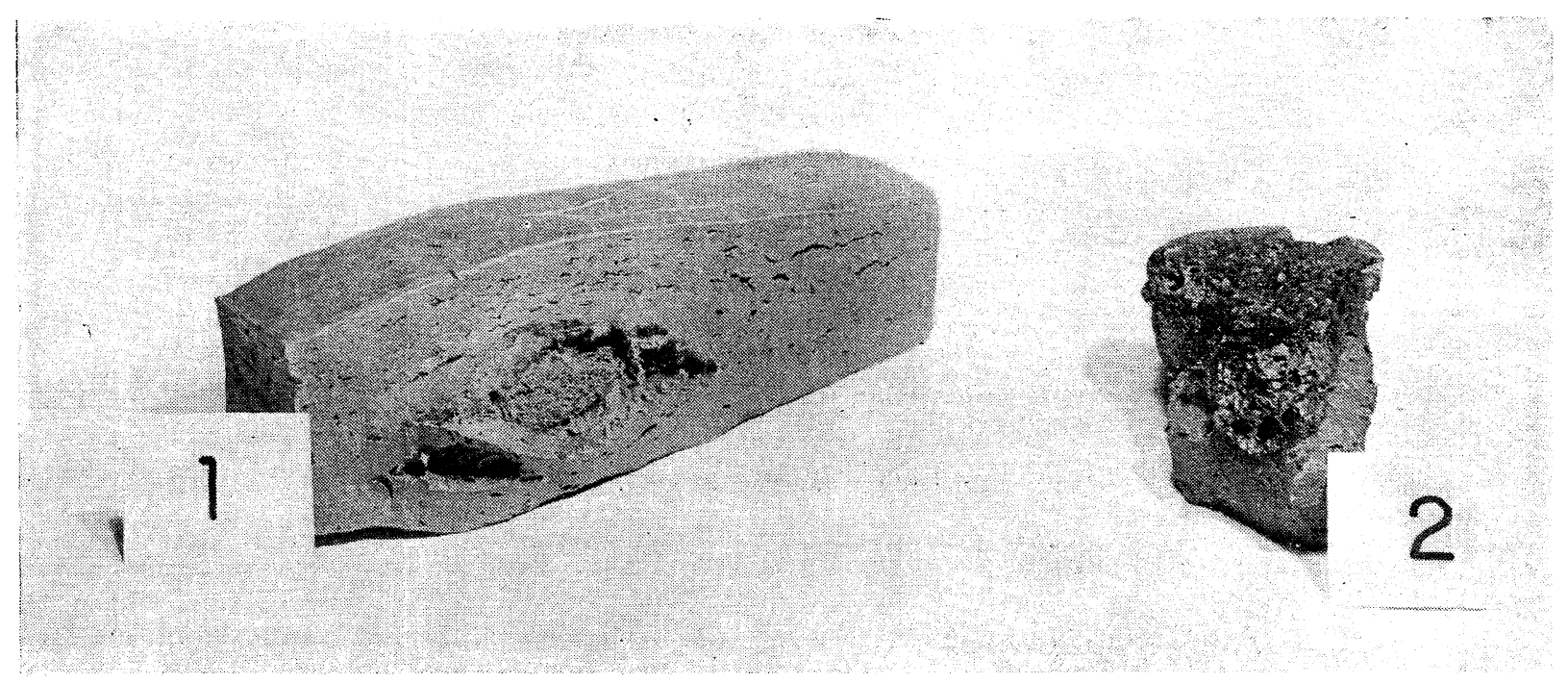

Fig, 2,-1. Aspecto típico de rotura por afloramiento de gas. 2. Corte mostrando el núcleo en descomposición.

6.4. En ciertos casos se desea una coloración final que no es posible sin la colaboración del óxido férrico y, sin embargo, la temperatura de cocción es elevada. Es preciso en este caso proceder a una reoxidación a temperaturas inferiores a las de descomposición. Este proceso es lento y se puede controlar por el aumento de densidad de los productos cocidos.

Se pueden conseguir ladrillos blancos con un contenido de $0,5 \%$ de hierro, evitando esta reoxidación.

6.5. En la cocción de los ladrillos de construcción no suelen alcanzarse las temperaturas de fusión de los eutécticos posibles en la mezcla de óxidos que se encuentran normalmente en las arcillas. La vitrificación produce una notable mejora de las características técnicas, pero alcanzar la temperatura necesaria resulta antieconómico en muchos casos. Las reacciones en fase líquida son poco frecuentes y las que se producen suelen ser consecuencia de la presencia de compuestos de bajo p.f., más que de lo elevado de la temperatura; un ejemplo es la formación del vidrio ferroso verdi-negro a la que hacíamos referencia antes. Otras veces los alcalinos actúan de fundentes y pueden producir una solución vitrea de hematita, de color amarillo, que en algunos ladrillos puede observarse en los intersticios.

\section{7. agentes colorantes menos frecuentes}

A pesar de todos estos estudios y de que normalmente se atribuye el color rojo de los ladrillos a la presencia de hematita libre, Salmang cita el caso de arcillas que cuecen rojo en ausencia de hierro, encontrándose en el producto cocido cantidades de $\mathrm{SO}_{3}$ que oscilan del 8,49 al 19,58\%. Vetter (5), que cita estas experiencias, explica el color por la formación de sulfoferritos que dan coloraciones intensas con cantidades minimas de hierro. 
Este fenómeno puede ser debido a otras causas. Se ha explicado el desarrollo del color por la formación de cadenas de polisulfuros, las cuales presentan coloraciones rojas que se oscurecen a medida que la cadena se alarga. Estos polisulfuros se forman a partir de los sulfuros de hierro o de manganeso, pero en último caso el responsable del color es la agrupación ...-s-s-... y no el catión correspondiente. No es preciso tampoco que los sulfuros se encuentren en la materia prima; impurificaciones procedentes de los humos pueden aparecer en el curso de la cocción dando tonalidades, o manchas de color, distintas según las condiciones de cada caso.

Los sulfuros pueden llevar impurezas de seleniuros, capaces de reducirse por acción del $\mathrm{SO}_{2}$ a selenio, en estado metaloideo, que tiene una brillante coloración roja, y que puede producir en el ladrillo manchas más o menos grandes o tonalidades de color rojizo.

\section{8. influencias externas}

8.1. En el siguiente ejemplo de Hedin se aprecia perfectamente la influencia de la temperatura: los datos corresponden a los colores de cocción de una misma arcilla.

$\begin{array}{rrrl}900^{\circ} & \mathrm{C} & \ldots \ldots \ldots \ldots \ldots \ldots . . & \text { Rojo amarillento } \\ 1.000^{\circ} & \mathrm{C} & \ldots \ldots \ldots \ldots \ldots \ldots \ldots & \text { Amarillo rojizo } \\ 1.050^{\circ} & \mathrm{C} & \ldots \ldots \ldots \ldots \ldots \ldots . & \text { Amarillo claro } \\ 1.100^{\circ} & \mathrm{C} & \ldots \ldots \ldots \ldots \ldots \ldots \ldots & \text { Verde amarillento }\end{array}$

El primer color es el normal producido en la sinterización. Los dos colores amarillos pueden proceder de una misma reacción en dos grados de desarrollo, del tipo de una solución vítrea de hematita, o de una descomposición. Habría que estudiar el material para dar una opinión menos aventurada. El color verde amarillento ya es típico de los vidrios ferrosos, que también pueden producirse a elevada temperatura, por descomposición parcial del óxido férrico y fusión de algún silicato.

Por último, el desarrollo de las reacciones será más intenso cuanto más homogénea sea la mezcla. Citamos a continuación otro ejemplo tomado del trabajo de Hedin:

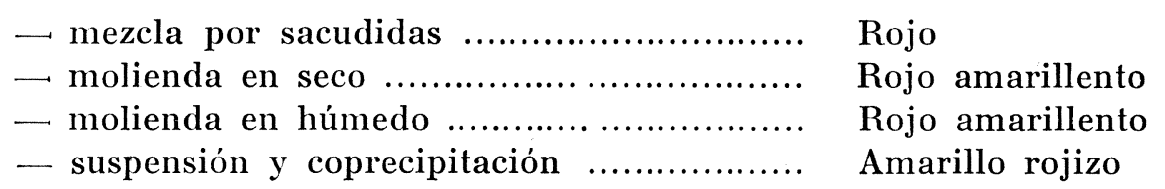

Todos los colores corresponden a una temperatura de cocción de $1.000^{\circ} \mathrm{C}$ y los cambios de color pueden interpretarse como la consecuencia de la formación de mayor cantidad de compuestos verdaderos.

\section{9. conclusión}

A pesar de lo numeroso de las reacciones posibles, en la práctica sólo se desarrollan muy pocas en cada caso. Los defectos de coloración son corregibles; y la corrección del color posible, en la mayoría de los casos, aunque no siempre aconsejable.

El problema de la corrección del color es fundamentalmente económico; no son aconsejables las adiciones de tipo químico, y sí la mezcla de diversas arcillas que compensen las deficiencias.

La corrección de los defectos será siempre la consecuencia de un detenido estudio de las materias primas y del proceso de elaboración. 


\section{bilbiliografina}

Clates Hedin: The colour of clay products after burning. The British clayworker, noviembre 1954.

Grewe: Der Ton und seine Brennungsfärbung. Keramische Zeitschrift, 1953, núm. 12.

O.F.: Sul colore dei laterizi. L’Industia italiana dei laterizi, diciembre 1954.

Kenneth Shaw: Ceramics colors ... here's a new approach. Ceramic Industry, agosto 1962, vol. 79, pág. 42.

H. Vetter: Ueber die färbenden Bestandteile von Tongesteinen und ihr Brennverhalten. Die Ziegelindustrie, 1954, núms. 19 y 20.

The Am. Ceram. Soc. Ind., 1964. Phase diagrams for ceramists.

R. WeSt: Colouration of brick manufactured from limey clays. J. Canad. Ceram. Soc., 1962, 31, 94-9.

Searle: An Enciclopedia of the ceramic industry. Ernest Benn, Londres, 1929.

JAckson: Oxidation of ceramic ware during firing (7 articulos). Bull. Am. Ceram. Soc., 14 (7), $225,1935$.

Anónimo: Iron compounds in the ceramic industry. Transaction of the British Ceramic Society, octubre 1951.

Gilbert C. Robinson y L. H. Wright: Reduction in oxidation time through changes in body composition. Am. Ceram. Soc. Bull., septiembre 1961, pág. 545.

Anónimo: They've reduced black coring with ammonium cloride. Brick \& Clay Record (126), 2, 1955.

Anónimo: Reactions accompanying the firing of bricks. J. Am. Ceram. Soc., 34 (3) 71-76, 1951.

Anónimo: Control kiln atmosphere for better brick colors. Brick \& Clay Record, septiembre 1959.

F. Detrmer: Ueber die Bedeutung des Eisenoxydes für den Brandausfall Keramischer Massen. Sprechs (17), 317, 1928.

KERL: Handbuch der gesamten Tonwarenindustrie. Vieweg und Sohn, Braunscheig, 1907.

RoBson: Operating the tunnel kiln. Ind. Pub., Inc. Chicago, 1954, págs. 22 y 114.

F. SANDFond \& B. Liljegren: The formation of colour in red and yellow bricks. Chalmers Tekniska Högakolas Handlinger, núm. 282, 1963.

Lawrence H. von Vlack: Physical Ceramics for engineers. Addison-Wesley Publ. Co. Inc., London, 1964. 